\title{
Visión de futuro y metodología para LA PRODUCCIÓN MODERNA DE NUEVO CONOCIMIENTO TECNOLÓGICO EN LOS POSGRADOS DE DISEÑO INDUSTRIAL DE MÉXICO
}

Future's Vision and methodology for the modern production of new technological knowledge of Industrial Design's Postgraduate Schools of Mexico

ERNESTO OcAMPo-RUIz* 2 Septiembre 2020 Fecha de aceptado: 19 Marzo 2021

RESUMEN. En un contexto contemporáneo mundial influenciado por el Modo 2 de Gibbons en la producción del conocimiento universitario, como frente a la baja evidencia histórica de productos tecnológicos operacionales y terminados transferidos con éxito y de forma mediata a la sociedad provenientes de la Disciplina del Diseño Industrial, así como ante los nuevos retos transdisciplinarios que enfrentan los posgrados de diseño industrial de las IES mexicanas en la necesidad del desarrollo de tecnología útil, madura, competitiva, inmediatamente aplicable y de calidad confirmada mediante normatividad, se ofrece como camino alternativo supra metodológico de I\&D+D, la introducción en la planeación escolar de seminarios y talleres del área tecnológica del diseño del TRL: Technology Readiness Level en la estructura pedagógica y didáctica de los posgrados de diseño industrial mexicanos, instrumentado a partir de la experiencia aplicada y comprobada con resultados óptimos cuantificables, obtenidos durante las últimas dos décadas, en la producción tecnológica de vanguardia realizada en el Posgrado de Arquitectura de la unAm.

Palabras clave: Modo 2 de Gibbons, Producción del Conocimiento, Productos Tecnológicos de Diseño Industrial, Technology Readiness Level, Transferencia Tecnológica, Transdisciplina.
ABSTRACT. In a contemporary world context influenced by Gibbons' Mode 2 in the production of university knowledge, and given the low historical evidence of operational and finished technological products successfully and mediately transferred to society from the Industrial Design discipline, and faced with the new transdisciplinary challenges in the Mexican higher education institutions' industrial design postgraduates in the need to develop useful, mature, competitive, immediately applicable technology and of quality confirmed by regulations, it is offered as an alternative methodological super method of R\&D, by inserting in school planning of seminars and workshops in the design's technological area the use of TRL: Technology Readiness Level, inside the pedagogical and didactic structure of Mexican industrial design postgraduate schools, instrumented from the applied and verified experience with optimal quantifiable results, that was obtained during the last two decades, in the cuttingedge technological production carried out at the UNAM Architectural Postgraduate School.

Key words: Gibbons Mode 2, Knowledge Production, Industrial Design Technological Products, Technology Readiness Level, Technology Transfer, Transdiscipline. 


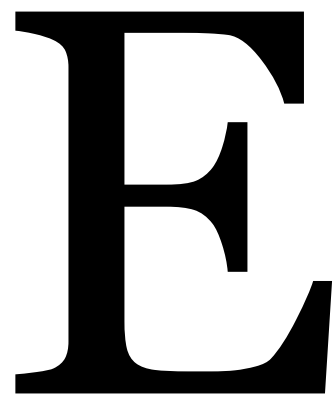

n las últimas décadas, la producción de conocimiento del Campo de Tecnología del Programa de Maestría en Diseño Industrial de la Universidad Nacional Autónoma de México (UNAM) no ha generado profusamente productos tecnológicos en congruencia a nuestra época. En el siglo xxi, la visión de futuro en la producción de conocimiento científico y tecnológico trasciende los métodos de investigación tradicionales y evolucionó a un nuevo modelo mundial, que busca la transdisciplina, como la definición clara de productos de impacto y potencial social con aplicabilidad inmediata. En la tecnología creada desde las Instituciones de Educación Superior (IES), como en la Educación de Posgrado, el Modo 2 de Gibbons establece un marco filosófico y procedimental claro y estable en la producción de tecnología universitaria, enriquecido por la aplicación del Método TRL: Technology Readiness Level para materializar, probar y transferir realmente tecnología de punta y emergente desde la universidad hacia la sociedad. De forma metodológica suprageneral, el TRL marca los pasos correctos para las etapas de $\mathrm{I}^{\mathrm{S}} \mathrm{D}+\mathrm{D}^{1}$ de productos tecnológicos universitarios, al mismo tiempo que establece un nivel de madurez normalizado y equiparable global. Para el Diseño Industrial, la herramienta pone orden operacional a los procesos y métodos habituales del diseño (Archer, Buchanan, Brown, Cross, Jones, etc.), como al Método Científico, para generar tecnología madura y de calidad (Latorre Cosculluela, 2020: 1-13).

1 Por sus siglas universalmente reconocidas I\&D+D, Investigación y Desarrollo (Tecnológico) más Diseño. Forma de generar conocimiento y productos tecnológicos que requiere durante sus etapas de desarrollo de métodos de diseño para madurar y perfeccionar sus productos entregables a la sociedad. 


\section{LA PRODUCCIÓN TECNOLÓGICA EN EL DISEÑO}

En las últimas décadas, la producción de conocimiento del Campo de Tecnología del Programa de Maestría en Diseño Industrial de la UNAM no ha llegado a generar profusamente productos tecnológicos contundentes en congruencia a nuestra época. Y no es un fenómeno aislado dentro de la integridad de la Disciplina del Diseño Mexicano, pues es un sesgo disciplinario metodológico generalizado que afecta lo que hoy se produce como tecnología para el diseño industrial desde las IEs.

El Programa, que es el caso de estudio y aplicación del que parte este análisis, está estructurado de acuerdo con su Plan de Estudios vigente en tres campos de conocimiento: Teoría e Historia, Ergonomía, y Tecnología. En los últimos veinte años, si bien han existido numerosos alumnos que han desarrollado sus tesis en el campo de tecnología, la producción del conocimiento generado por el Programa en la rama tecnológica no ha logrado cristalizar la generación recurrente de patentes tecnológicas, modelos de utilidad o diseños industriales registrados ante el Instituto Mexicano de la Propiedad Industrial en los últimos diez años (López, 25 de octubre de 2018, 1-3).

Efectuar el trámite, tanto dela patente como del modelo de utilidad, así como registrar el diseño industrial tiene congruencia como paso necesario posterior al modo de creación del conocimiento tecnológico que realizan las universidades para poder transferir la tecnología recién creada o innovada finalmente a la sociedad que la necesita.

\footnotetext{
Registrar el derecho de autor de las tecnologías desarrolladas en las universidades, es una tendencia generada desde la década de los años noventa del siglo xx a nivel mundial, y que es aceptada globalmente como producto de investigación final válido y cuantificable por organismos encargados de vigilar $\mathrm{y}$ promover las actividades científicas y tecnológicas a nivel mundial, como lo es en México el Consejo Nacional de Ciencia y Tecnología
}

(CONACYT), que lo utiliza como indicador de producción para la evaluación de la calidad ${ }^{3}$ de los Programas de Posgrado Universitarios, impartidos en las IEs en el país, trámite que ayuda a confirmar su productividad tecnológica como su trascendencia social y económica durante su operación.

Para el desarrollo de la tecnología, esta modalidad de transferencia tecnológica hoy presente en las IES en todo el mundo, y que le da importancia a la patente, es de hecho la manera en que se registra formalmente, y de manera correcta el hacer exitoso del diseñador industrial, del científico o del tecnólogo, como también se verifica la presencia ante el público de un producto confirmado, creativo y novedoso en ciernes, y que será útil para atender las necesidades de la sociedad, cuando éste sea impulsado y detallado para llegar al mercado comercial aprovechando los canales formales y habituales de la economía mundial (Casas Guerrero, 2000: 25-48). Esta es una de las formas más efectivas que hoy se manejan para vincular el conocimiento tecnológico desarrollado en las IEs con los medios de producción.

El registro de autoría es importante porque permite además que las universidades e IES protejan su valiosa inversión (de origen público o privado) en la formación de recursos humanos profesionales y especializados, como en los ingeniosos productos de investigación y desarrollo tecnológico (I\&D), que ellos realizan durante sus estudios de posgrado y que tienen utilidad práctica pública, como un valor económico y social para la comunidad, y sobre todo el medio empresarial que busca estas ideas nuevas para explotarlas bajo licencia comercial.

Es por ello, que se vuelve estratégico para el Posgrado de Diseño Industrial (PDI) de la UNAM, al menos desde el punto de vista de la calidad educativa que ofrece, generar tecnología en objetos creativos, innovadores y hasta disruptivos, que esté al nivel de desarrollo requerido, suficientemente madura, para poder ser patentable al final del tiempo escolar que los alumnos cursan para obtener su grado académico.

\footnotetext{
2 La UNAM logró 43 patentes en 2017, que fueron generadas por 178 investigadores de la UNAM, abarcando las áreas de energía (petróleo y energías renovables), industria aeroespacial, compuestos farmacéuticos, dispositivos médicos, sistemas electrónicos, alimentos, nuevos materiales y tratamiento de aguas residuales, ninguna proveniente del Posgrado de Diseño Industrial (López, 30 de abril de 2018).
}

\footnotetext{
3 "Se obtienen una pluralidad de productos [En los programas de posgrado] de la investigación y desarrollo, como publicaciones académicas, informes técnicos, patentes, prototipos y procesos, de carácter innovador, que demuestran originalidad y pensamiento creativo que reestructura la visión de los problemas en el campo"..." [Como medio de verificación]. Evidencia de la productividad (artículos, libros, participación en congresos nacionales e internacionales, desarrollos tecnológicos, patentes, derechos de autor, desarrollo de software, etc.) de los estudiantes según la orientación del programa2 (CONACYT, 2015: 28, 47, 50 y 51).
} 


\section{LA TRANSDISCIPLINA EN EL MODO DE CREAR CONOCIMIENTO CIENTFFICO Y TECNOLOOGICO EN EL SIGLOXXI}

Hoy ya no se hace ciencia y tecnología como se hacía antes. Al menos no tiene el mismo derrotero y alcances que se le comprendían antes. El modo hoy imperante de producción de conocimiento científico y tecnológico es nuevo y se gesta a partir de un cambio radical de visión sobre el hacer de la ciencia y de la tecnología nacido recientemente, y que es diferente al modelo que imperó durante casi todo el siglo xx de manera global.

En los albores del siglo xxi, la visión de futuro en la producción de conocimiento científico y tecnológico trasciende los métodos de investigación tradicionales y evolucionó a un nuevo modelo mundial, que busca la transdisciplina, como la definición clara de productos de impacto y potencial social con aplicabilidad inmediata al término del proceso mismo invertido en el desarrollo de la investigación (Gibbons, 1997: 72-74).

El profesor Michael Gibbons y su equipo de la Universidad de Sussex, y colaboradores de la Unión Europea, acuñan en 1994 el concepto del "Modo 2", que es un término desde la sociología de la ciencia que se refiere a la forma en que actualmente se produce el conocimiento (científico y tecnológico). Contrasta con el "Modo 1" de producción de conocimiento, que se le ha asignado a la forma tradicional intradisciplinaria. Esta definición impacta a nivel mundial, generando un movimiento internacional que lleva a la organización del World Conference of Science for the 21st Century: A new Commitment, que se realizó en Budapest, Hungría por la Organización de las Naciones Unidas para la Educación, la Ciencia y la Cultura (uNESCO, por sus siglas en inglés) en 1999. De los resultados de esta conferencia, surgen acuerdos para financiamiento $\mathrm{y}$ enfoques de visión futura en el hacer científico y tecnológico, dirigido a la docencia en las IEs; acuerdos que finalmente son orquestados por la Organización de las Naciones Unidas (ONU) y sus países agremiados. México no se queda fuera de este movimiento mundial y se adhiere a estos compromisos (UNESCO, 1999: 1-20).

En el Modo 1, o modo tradicional de hacer ciencia (Sañudo Guerra, 2017: 1-29), la iniciativa de investigar es personal, desde el investigador, por curiosidad. El problema por estudiar se define desde el contexto de interés esencialmente académico. En el campo de conocimiento de la investigación es esencialmente disciplinario, y en colaboración alcanza a ser interdisciplinario o multidisciplinario, mientras que el tipo de visión y respuestas son homogéneos provenientes de una sola visión disciplinaria. La organización del trabajo del investigador con su grupo de trabajo es jerárquica o piramidal, y la colaboración sucede entre pares especialistas o por Institución (figura 1).

El método de investigación es decidido lineal y tradicionalmente desde cada disciplina, mientras que el investigador se hace responsable principalmente con la innovación y la generación de conocimiento,
Para la disciplina de origen,

el impacto final del

conocimiento generado

ESTÁ ENCAPSULADO MONODISCIPLINARIAMENTE, Y REGRESA A LAS DISCIPLINAS DE ORIGEN COMO PRUEBA Y CALIBRACIÓN DEL PARADIGMA EXISTENTE, DONDE NO HAY APROPIACIÓN DE CONOCIMIENTOSO MÉTODOS

QUUE SON AJENOS A

LA DISCIPLINA ORIGINAL.

con un impacto social ex post, se le conoce o dimensiona solo cuando los resultados son divulgados, y/o aplicados por otras disciplinas posteriormente, tal vez mucho tiempo después. El financiamiento es Institucional, Sectorial, Interinstitucional o Intersectorial. Su evaluación es realizada por Pares de la Comunidad Científica, donde se revisa la calidad de la Construcción del Conocimiento y su fortaleza teórica. Para la disciplina de origen, el impacto final del conocimiento generado está encapsulado monodisciplinariamente, y regresa a las disciplinas de origen como prueba y calibración del paradigma existente, donde no hay apropiación de conocimientos o métodos que son ajenos a la disciplina original.

En contraste, en el Modo 2 la iniciativa de comenzar una investigación surge por necesidad apremiante, de arriba hacia abajo o de abajo hacia arriba, mientras que la definición del problema es desde el contexto de la aplicación mediata, es decir, que debe tener aplicación inmediata para la sociedad a la terminación y entrega del producto del proyecto. Su campo de conocimiento es transdisciplinario y hasta pluridisciplinario. El tipo de investigación es heterogénea, usando un método de investigación heterárquica, con toma de decisiones horizontal. El proyecto existe en redes interconectadas, de colaboración especializada y social. 


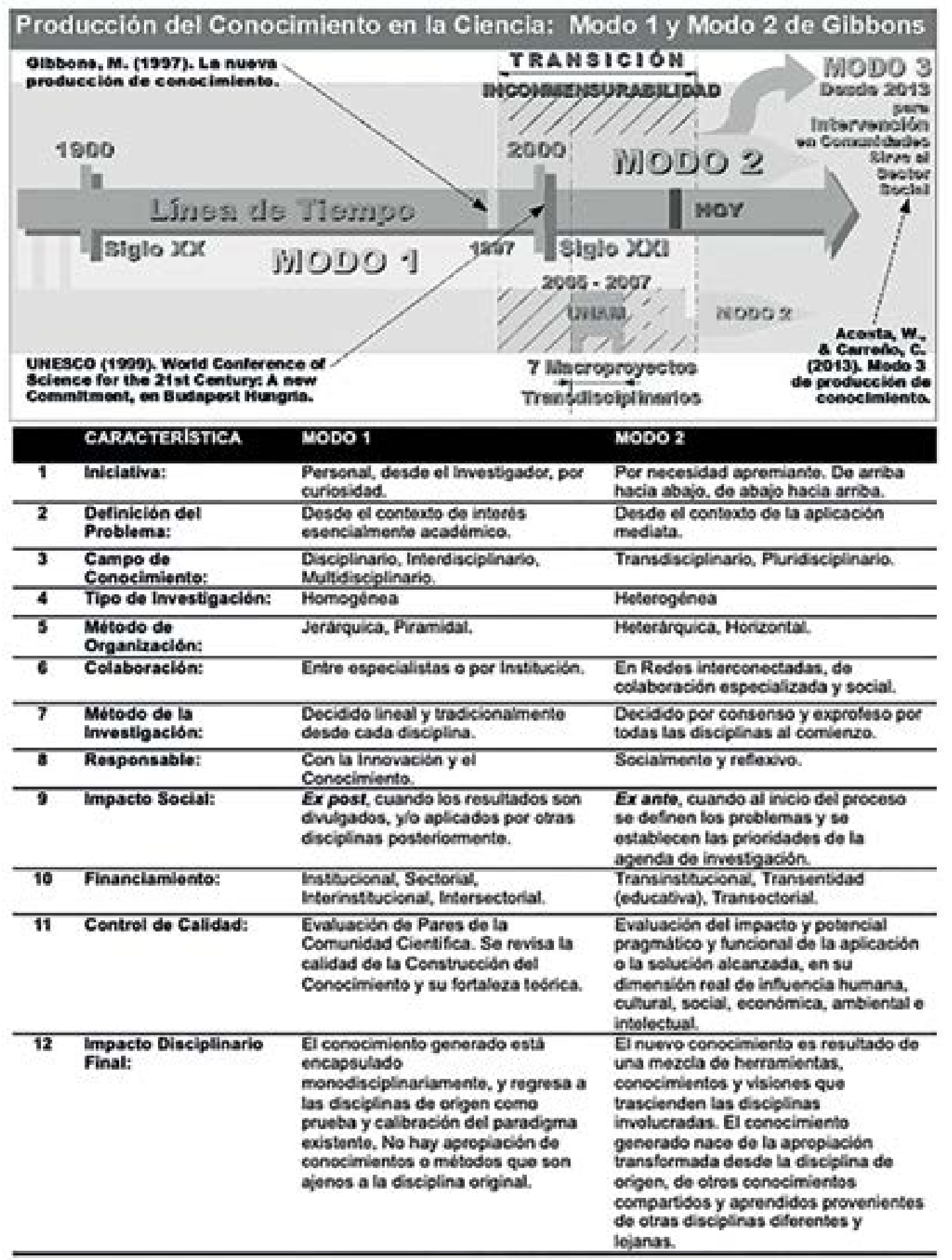

FIGURA 1. MODOS DE PRODUCCIÓN DE CONOCIMIENTO. EL MODO 1,2 Y 3 EN LA LÍNEA DEL TIEMPO CON LOS MACROPROYECTOS REALIZADOS EN LA unam. SE OBSERVA CLARAMENTE EL MOMENTO EN EL MUNDO DEL CAMBIO DE PARADIGMA HACIA EL MODO 2 DE GIBBONS, MIENTRAS LA unam PROPCIIA LA VISIÓN INTERNA DE LA INSTITUCIÓN HACIA LA TRANSDISCIPLINA COMO SU CONSECUENCIA, A TRAVÉS DEL PROGRAMA TRANSDISCIPLINARIO EN INVESTIGACIÓN Y DESARROLLO PARA FACULTADES Y ESCUELAS, CON SUS SIETE MACROPROYECTOS TRANSDISCIPLINARIOS. CON LA TABLA SE COMPARAN LAS DIFERENCIAS RADICALES ENTRE EL MODO 1 Y 2 EN SU ESENCIA.

FUENTE: ELABORACIÓN PROPIA COCAMPO RUIZ, 10-11DE JUNIO DE 2019: PONENCIA]. 
Los métodos de investigación son decididos por consenso y diseñados exprofeso por todas las disciplinas que participan en el proyecto al comienzo. Su responsabilidad trasciende al puro ejercicio de crear conocimiento, buscando ser socialmente responsable y reflexivo. Su impacto social es ex ante, y se le conoce cuando al inicio del proceso se definen los problemas y se establecen las prioridades de la agenda de investigación. El financiamiento es compartido por muchas instancias siendo transinstitucional, transectorial. El control de calidad final se hace mediante una evaluación del impacto y potencial pragmático y funcional de la aplicación o la solución alcanzada, en su dimensión real de influencia humana, cultural, social, económica, ambiental e intelectual. El impacto disciplinario final del nuevo conocimiento es resultado de una mezcla de herramientas, conocimientos $\mathrm{y}$ visiones que trascienden las disciplinas involucradas. El conocimiento generado nace de la apropiación transformada desde la disciplina de origen, de otros conocimientos compartidos y aprendidos provenientes de otras disciplinas diferentes y lejanas durante el trabajo de investigación. ${ }^{4}$

En la tecnología creada desde las IEs, como en la Educación de Posgrado, este nuevo modelo -el Modo 2- generó alrededor del planeta un cambio profundo que transforma los modelos educativos, como los procesos internos universitarios de organización, financiamiento, evaluación, gestación, investigación y desarrollo tecnológico (Didriksson, 2005: 95-125).

La UNAM quedó inmersa en este proceso y protagonizó un cambio radical en la forma de pensar y hacer la ciencia y la tecnología. De 2005 a 2007, la institución nacional creó el Programa Transdisciplinario en Investigación y Desarrollo para Facultades y Escuelas (PTIDFE) 5 dirigido a transformar hacia la investigación transdisciplinaria, de manera amplia y masiva, la forma en que la UNAM realizaba la investigación científica y tecnológica. El programa abrigó siete macroproyectos institucionales que contaron con la colaboración y apoyo de 23 facultades y escuelas, 17 centros e institutos,

\footnotetext{
4 Existe un Modo 3, definido por el equipo del investigador Wilson Acosta Valdeleón de la Universidad La Salle en Bogotá, y es una modificación o variante del Modo 2, pero totalmente etnográfico, que subraya una participación superlativa de la comunidad, por encima de los investigadores, acerca de qué investigar, cómo investigar, y para qué investigar. Las decisiones sobre un proceso de investigación las toma una comunidad, no el equipo de investigadores, incluyendo el método (Roa-Mendoza, 2016: 106-110).
}

5 cfr. Acuerdo por el que se crea el Programa Transdisciplinario en Investigación y Desarrollo para Facultades y Escuelas (Gaceta UNAM, 15 de agosto de 2005, p. 24). e instituciones como el Instituto Politécnico Nacional. En este esfuerzo de transformación hacia el Modo 2, fueron convocados y laboraron más de un millar de participantes, entre profesores, investigadores, como alumnos de la institución. Para los productos de origen transentidad educativa como transdisciplinarios en su hacer, se crearon comités editoriales, comités académicos, métodos de trabajo y producción exprofeso y formas de evaluación con pares académicos y expertos internacionales. Los siete macroproyectos trabajaron con temáticas de interés nacional, de impacto social, y de transformación para la misma institución nacional. Hoy la UNAM, trabaja la investigación científica y tecnológica, principalmente en el Modo 2, de forma transdisciplinaria, como también lo hace la mayor parte de las IEs en la nación, gracias a este esfuerzo institucional. Un esfuerzo que ha impactado también el espíritu y la calidad de cada Plan de Estudios a nivel profesional y de posgrado en toda la UNAM (Estruch Tobella, 2002: 86-88).

Para los que participamos directamente en la realización de los Macroproyectos, coordinando desde la cúpula académica y administrativa del programa, hubo grandes aprendizajes. Enfrentarse a una tarea universitaria, así desde la Rectoría de la UNAM, contemplando a vuelo de pájaro la integridad de la Institución y viviendo toda la magnitud de sus instalaciones universitarias a nivel nacional, percibiendo la dimensión de su infraestructura y su gente, como conviviendo e interactuando con la gran diversidad de visiones y procesos de trabajo divergentes de cada uno del millar de investigadores y participantes que trabajaron en los siete macroproyectos del PTIDFE, hace posible al final del trabajo entregado que la visión personal se sitúe por encima y lejos de lo aprendido en la propia disciplina de origen, y se comprenda allanando caminos como disolviendo obstáculos, la inconmensurabilidad natural presente entre tantas disciplinas disímbolas, incluyendo la propia. Permite ver al Diseño Industrial como a otras disciplinas del diseño, desde afuera, desde arriba, rompiendo con lo propio y entendiendo finalmente la transdisciplina.

Lo aprendido desde la transdisciplina en esta experiencia fue determinante e ilustrativo:

1. La producción de conocimiento útil y que trasciende es una labor compartida de índole transdisciplinaria que debe reunir expertos de disciplinas diferentes 
para acercarse a una solución que atiende integralmente la complejidad de un reto real. Las soluciones intradisciplinarias son parciales y cortas en alcance porque no ven el fenómeno multidimensionalmente.

2. En la tecnología creada desde las IEs, como en la Educación de Posgrado, el modelo transdisciplinario (Modo 2 de Gibbons) generó alrededor del planeta un cambio profundo que transforma los modelos educativos, como los procesos internos universitarios de organización, financiamiento, evaluación, gestación, investigación y desarrollo tecnológico.

3. Que, en países como México, no acostumbrados culturalmente a desarrollar tecnología de punta y patentes, existe el malentendido de usar sólo el Método Científico para producir tecnología en las IEs, provocando que los proyectos tecnológicos no maduren para ser transferidos a la sociedad y sean evaluados por pares que no tienen la experiencia de hacer realmente tecnología, sino ciencia.

\section{EL USO METODOLÓGICO DEL TRL 0 NIVEL DE MADUREZ DE TECNOLOGÍA EN EL MODO DE PRODUCCIÓN DE CONOCIMIENTO TECNOLÓGICO UNIVERSITARIO}

Si bien, el Modo 2 de Gibbons (1997: 51-63), como se conoce a esta forma contemporánea de producir conocimiento científico y tecnológico, establece un marco filosófico y procedimental claroy estable en la producción de tecnología universitaria, ésta se ve enriquecida globalmente por la aplicación del Método TRL: Technology Readiness Level (CONACYT, 2014: 1-7) para materializar, probar y transferir realmente tecnología de punta y emergente desde la universidad hacia la sociedad.

Como toda disciplina cultural humana, la tecnología tiene su propio método para generar sus productos (figura 2). A diferencia de lo que se piensa, el método científico, hoy conocido como el método experimental de la ciencia, no ayuda en nada a organizar el desarrollo de un objeto tecnológico complejo paso a paso desde el surgimiento de la idea, pasando por la prueba de diversas etapas de perfeccionamiento de prototipos, hasta su entrega como piloto comercial primero y luego producto comercial. El método que en realidad se usa para generar tecnología en todo el mundo es propio de la disciplina de la tecnología exclusivamente y tiene un fundamento de visión y de planeación de tipo estratégica y prospectiva. En este proceso único de la tecnología, el método científico ayuda a diseñar solamente los espacios de experimentación con los que son probadas las diferentes etapas de maduración de los prototipos, pilotos y productos. El método científico no permite la planeación de todo el proceso necesario para crear un objeto tecnológico, porque en realidad solo está diseñado para planear un experimento que ha de tener lugar. El método científico, como la ciencia en su dimensión de disciplina cultural humana, están en esta materia, en el caso de la I\&D+D, subordinados a lo que la tecnología hace y necesita. Son ambos, la ciencia y su método, para la tecnología sus herramientas subordinadas en el proceso.

Desde la antigüedad, el método para construir objetos tecnológicos y perfeccionarlos ha ido evolucionando para controlar el proceso y mejorar el resultado. En 1969, D.G. Marquis y S. Myers delinearon con precisión el método llamado "Desarrollo de Paquetes de Innovación Tecnológica" para hacer visible y claro el proceso paso a paso, tanto en la industria como en las IES, del Desarrollo Tecnológico de un objeto entregable a la sociedad (Cadena, 1986: 27-48). Años después, este método arrojó frutos y fue perfeccionado para la NASA por S. Sadin y R. Chase en 1995. Sadin lo llamó TRL o el "Método de Nivel de Madurez de la Tecnología” (Technology Readiness Level), y sirvió desde entonces para desarrollar y perfeccionar por etapas los equipos y naves de los programas espaciales de exploración que hoy conocemos (The United States Government Accountability Office, agosto de 2016: 16-25).

El método TRL, que tiene nueve niveles universales de maduración y sirve también para clasificar el nivel de avance en el desarrollo de un producto (The Federal Highway Administration, 2017: 19-20), fue rápidamente apropiado por toda empresa, industria u organismo dedicado a desarrollar tecnología a nivel mundial, y ha permeado desde entonces en Universidades e IES a nivel internacional para enseñar a hacer tecnología, como para generar objetos tecnológicos derivados de la producción de conocimiento que generan los alumnos y los investigadores de cada institución. Ha probado aclarar el proceso de I\&D+D como

\footnotetext{
6 Laborando en la Rectoría de la UNAM desde la UAIFE, cfr. Acuerdo por el que se crea la Unidad de Apoyo a la Investigación en Facultades y Escuelas (Gaceta UNAM, 15 de agosto de 2005, p. 25)
} 
ayudado a construir prototipos plenamente operacionales, útiles para la sociedad, probados en laboratorios y patentables en el corto tiempo (dos a tres años) que permite obtener un grado académico o profesional en cualquier universidad. ${ }^{7}$

El método TRL ya ha sido utilizado y probado con éxito en la formación de arquitectos tecnólogos, a nivel de maestría con resultados palpables desde 2005, en el Posgrado de Arquitectura de nuestra universidad. La maestría en arquitectura campo de conocimiento de tecnologías cambió al comenzar el siglo xxi, el enfoque que prevaleció de origen hace 45 años, en la selección de temas, determinación de los contenidos como en la realización de la investigación de las tesis de los alumnos a graduarse. Hasta 1999, las tesis producidas eran evidentemente monográficas, enciclopédicas, de sentido teórico y evidentemente basadas en compilaciones de simples investigaciones documentales, con poca disertación personal. Las aportaciones eran poco evidentes y algunas veces cuestionables desde el punto de vista de producción de conocimiento nuevo en el área de tecnología. Esta orientación en la evaluación de las tesis, acompañada de una latente y creciente ausencia de aplicabilidad de los productos terminales en la vida profesional de los alumnos al terminar vino acompañada de fuerte deserción, baja eficiencia terminal, desánimo estudiantil, y pocos productos memorables históricamente.

A partir de 1999, el campo de conocimiento de tecnología de la maestría en arquitectura buscó crear verdaderos objetos tecnológicos probados en laboratorio y con aplicabilidad inmediata para la sociedad. En otras palabras, el Campo de Tecnología decidió hacer tecnología arquitectónica real, pertinente y necesaria para la comunidad. Esta inmersión al Modo 2 de Gibbons fue acompañada años después, a partir de 2005 con la inclusión en los Talleres de Investigación del Método TRL

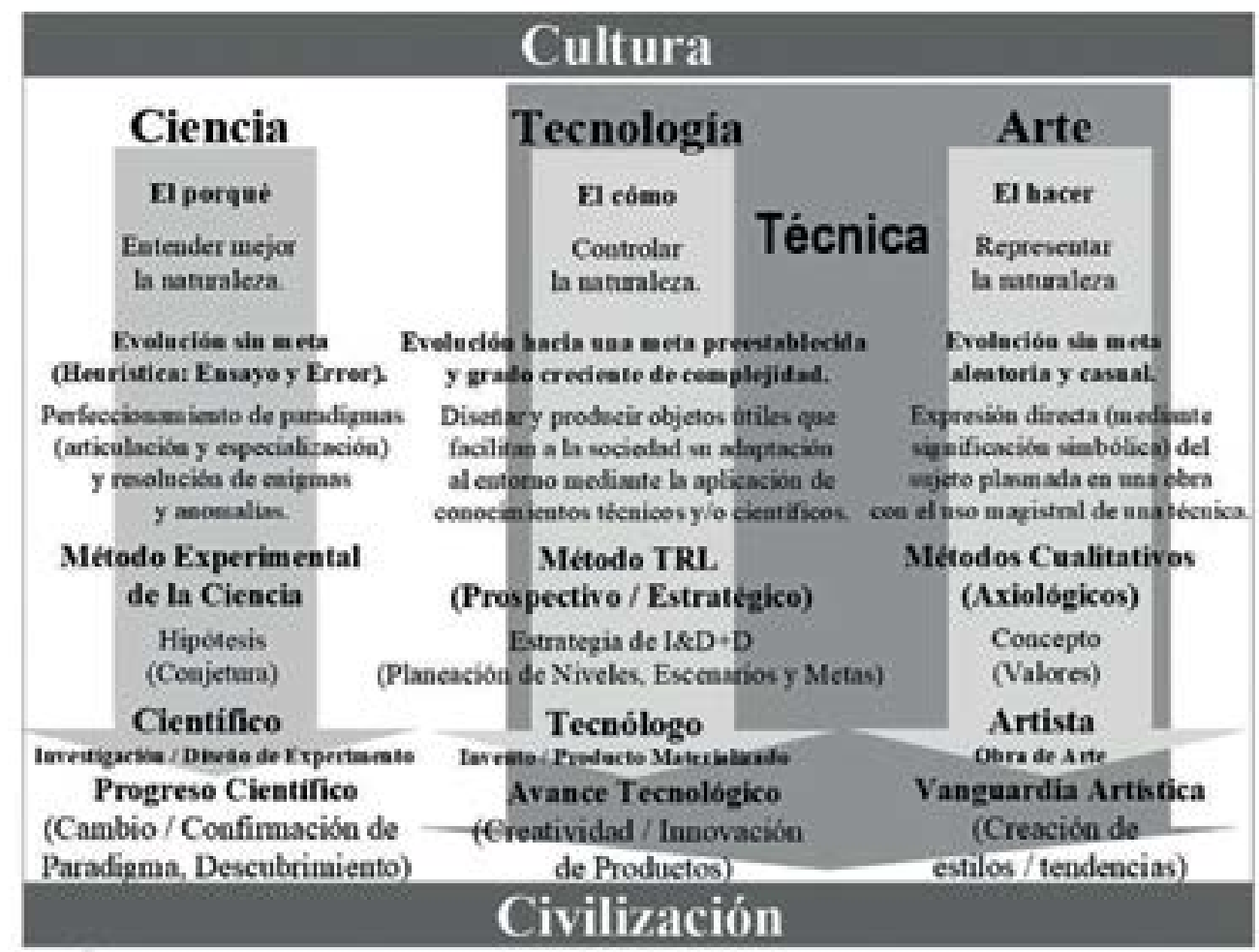

FIGURA 2. LA DIFERENCIA ENTRE TRES DE LAS DISCIPLINAS CULTURALES HUMANAS AFINES AL DISEÑO. LAS CARACTERÍSTICAS DE LA TECNOLOGÍA QUEE LA HACEN DIFERENTE DE LA CIENCIA, COMO DISCIPLINAS CULTURALES HUMANAS DE DIFERENTE ORIGEN, CON DIFERENTE PROPÓSITO, DE DIFERENTES PRODUCTOS. TIENEN, POR LO TANTO, DIFERENTES

MÉTODOS DE PRODUCCIÓN DE SU CONOCIMIENTO. EL MÉTODO CIENTFFICO, HOY CONOCIDO COMO MÉTODO EXPERIMENTAL ES INHERENTE Y ÚTL PARA LA CIENCIA EN SU PRODUCCIÓN, PERO EN LA TECNOLOGIA, EL TRL: TECHNOLOGY READINESS LEVEL ES EL PROCESO METODOLÓGICO CON EL QUE SE DESARROLLAN SUS PRODUCTOS. CADA DISCIPLINA TIENE FINALMENTE SU PROPIO MÉTODO.

FUENTE: ELABORACIÓN PROPIA COCAMPO RUIZ, 10-11DE JUNIO DE 2019: PONENCIAJ.

\begin{abstract}
7 A partir de 1999, los temas de investigación que distinguen a todos los alumnos del campo de tecnologías de la Maestría en Arquitectura de la UNAM cambiaron radicalmente e incluyen hoy el desarrollo de prototipos operacionales probados en laboratorios de institutos en las IES nacionales y extranjeras, con nuevos materiales arquitectónicos de alta tecnología (nanomateriales, composites avanzados, biomateriales), así como sistemas constructivos arquitectónicos para la eficiencia energética y ambiental, con la cosecha, uso y almacenaje de
\end{abstract}

recursos, tales como el agua o la energía alternativa sustentable, además de diseñar tecnologías pasivas, de incluir el reciclaje de polímeros en componentes constructivos prefabricados, o diseñar envolventes verdes o paramétricas, entre muchas otras tecnologías de punta y emergentes para la arquitectura. Pueden ser consultadas en TESIUNAM, que es el catálogo en línea en el cual se visualizan las tesis de los sustentantes que obtuvieron un grado académico o profesional en la UNAM (cfr. TESIUNAM, 26 de julio de 2019). 
en el desarrollo de tecnología arquitectónica de punta y emergente (figura 3). Hoy se desarrollan y entregan tesis que son el informe final de un proceso de I\&D+D que documenta la descripción del proceso de desarrollo tecnológico de las aplicaciones arquitectónicas, la reseña del proceso de fabricación de prototipos, con los resultados de las pruebas realizadas en laboratorios de nivel científico y alta calidad. Todos estos trabajos son impulsados a terminar, buscando alcanzar el Nivel 4 del TRL, que implica el desarrollo exitoso de un prototipo burdo probado en un ambiente de laboratorio completamente controlado, tal y como lo marca ese nivel de maduración en el método (Ocampo Ruiz, diciembre de 2019: 1-4).

Las consecuencias positivas y los amplios beneficios son ahora visibles, no hay deserción prácticamente, la eficiencia terminal por cohorte generacional es de $80 \%$, graduándose el resto de los alumnos de la generación en el siguiente semestre posterior al límite del cohorte, los alumnos viven entusiasmados, por lo que hacen $\mathrm{y}$ encuentra no solo aplicabilidad en su vida profesional sino potencial para generar un futuro mejor, donde el objeto tecnológico desarrollado les sirve como conducto para construir durante sus dos años de estudios, la especialidad que tendrán por el resto de su vida (Valle Flores, 2000: 19-20). La lista de tesis y las estadísticas del campo muestra la calidad que contrasta clara y favorablemente con el resultado existente de los otros campos que conforman el Posgrado de Arquitectura durante el mismo tiempo (Ocampo Ruiz, diciembre de 2019: 5-9).

\section{EL PAPEL DEL TRL O NIVEL DE MADUREZ DE TECNOLOGÍA EN EL DISENO INDUSTRIAL}

Aunque tradicionalmente el Posgrado de Diseño Industrial ha enfocado su esfuerzo académico a la teoría y discusión de la tecnología como también a promover el desarrollo de prospectos en etapa temprana de ideas que pueden llegar a ser interesantes productos de diseño para la sociedad, son pocos los ejemplos de tesis en los últimos veinte años que generan prototipos operacionales funcionando y probados en ambientes controlados de laboratorio para validar su rango de utilidad. Pocos de ellos eran buenos candidatos para llevarse a la siguiente etapa para entrega a la sociedad. Pocos de ellos fueron candidatos patentables, que finalmente no se registraron en su autoría intelectual.
El Posgrado de Diseño Industrial (PDI) de la UNAM entre 2005 y 2007, colaboró en dos desarrollos tecnológicos al cobijo del Programa Transdisciplinario en Investigación y Desarrollo para Facultades y Escuelas de la UNAM (PTIDFE). Tanto el automóvil multifuncional "Ecovía" impulsado por celdas de hidrógeno como la Luminaria Híbrida (eólica y fotovoltaica) del Macroproyecto 3 "La Ciudad Universitaria y la Energía", dirigido por la Facultad de Ingeniería, son posiblemente lo más avanzado en desarrollo de prototipos tecnológicos probados en laboratorio y puestos en marcha dentro del Campus Universitario que el Posgrado de Diseño industrial ha realizado bajo el Modo 2 en quince años. Así pasó con la Luminaria Híbrida que fue colocada en un espacio de exposición que la UNAM construyó entonces para verla funcionar junto a otras tecnologías generadas transdisciplinariamente por el PTIDFE.

Debemos ser claros, un campo de conocimiento de tecnología en cualquier programa de posgrado universitario debe generar nuevo conocimiento tecnológico útil y comprobado para su disciplina, y este conocimiento solo se puede construir haciendo, materializando, verdadera tecnología. Una buena teoría, que se derive del comportamiento de una tecnología, solo puede ser construida mediante la observación y la prueba de sí misma, materializada y caracterizada en laboratorios con equipos que permitan una observación científica de su comportamiento en la realidad, lo que implica la construcción de prototipos cada vez más complejos y la compra de equipos de medición. De la misma manera que solo un piloto aviador que vuela lo hace después de muchas horas de vuelo, quién hace y enseña cómo hacer tecnología requiere experiencia y práctica construyendo productos tecnológicos reales. Un agricultor no puede ser piloto aviador, ni un artista un médico. Se necesita vivir la experiencia de producir tecnología real para poder entender, realizar y evaluar proyectos de tecnología de punta plenamente operacionales.

Durante años, CONACYT aprobó, supervisó y evalúo tecnología con pares provenientes de la ciencia teórica y no aplicada, usando el método científico para calificar los resultados. Esto fue siempre contradictorio y polémico. Cada disciplina cultural humana, como la ciencia, la tecnología y el arte, tienen en

\footnotetext{
8 Cuyo responsable académico del desarrollo tecnológico de ambos proyectos ante el PTIDFE fue el Dr. Oscar Salinas Flores, Coordinador del Programa de Maestría en Diseño Industrial de la UNAM, en ese entonces (cfr. Unidad de Apoyo a la Investigación para Facultades y Escuelas, 2007, pp. 3-6).
} 


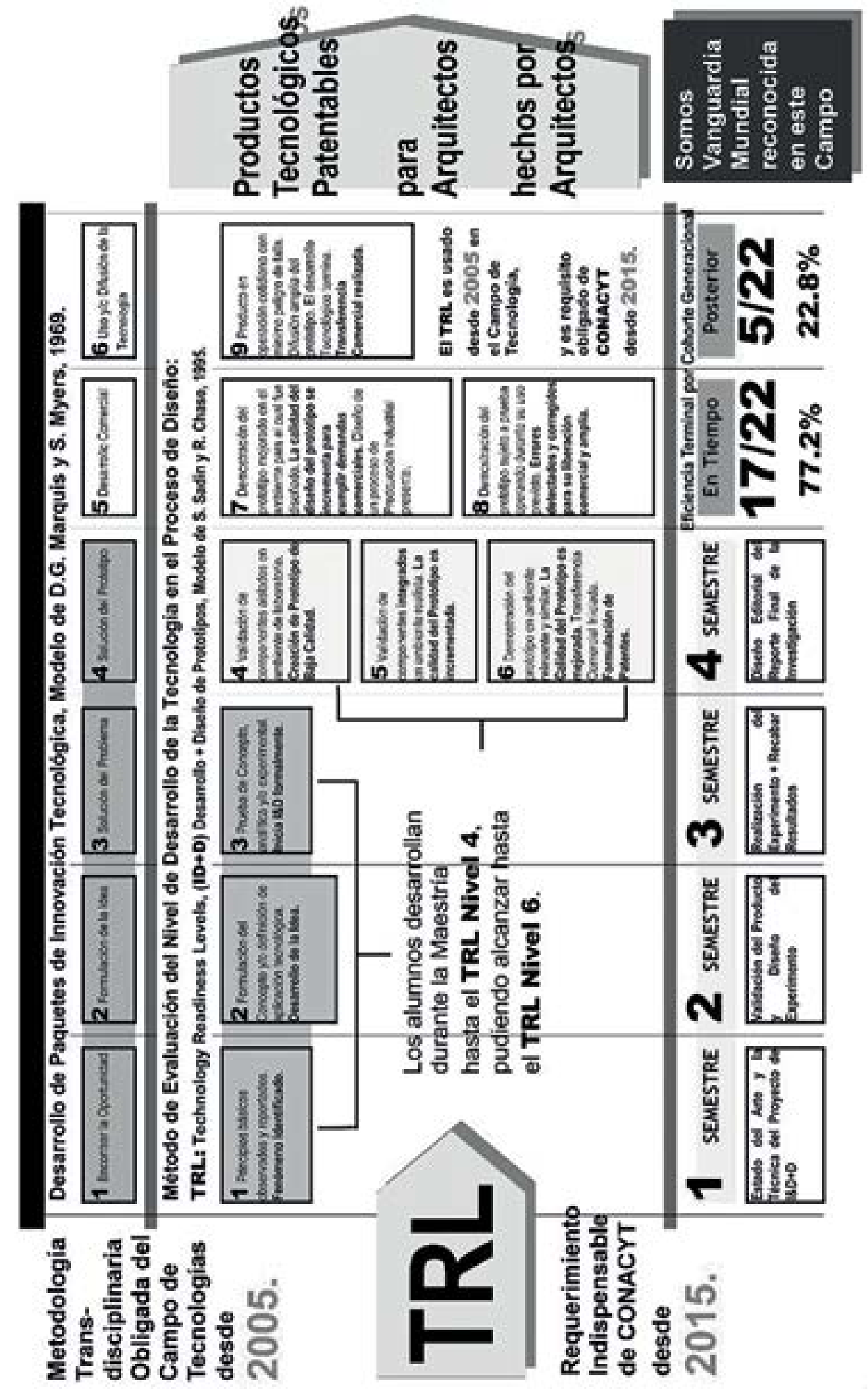

FIGURA 3.TRL APLICADO AL POSGRADO DE ARQUUTECTURA. EL TRL INFLUYE CAMBIANDO EL ALCANCE, TANTO DE LOS ALUMNOS COMO DE LOS PRODUCTOS PARA BIEN DE TODOS, ELEVANDO LA CALIDAD EDUCATIVA DEL CAMPO DE CONOCIMIENTO DE TECNOLOGíAS EN LA MAESTRÍA EN ARQUUTECTURA DE LA unam. SE OBSERVA DE MANERA VERTICAL, CÓMO SE TRANSFORMAN Y APLICAN CORRELACIONÁNDOSE, TANTO EL MÉTODO DE PAQUETE TECNOLÓGICOS DE D.G. MARQUIS COMO EL TRL: TECHNOLOGY READINESS LEVEL DE S. SADIN, CON LA ESTRUCTURA DE LOS SEMESTRES QUE SE IMPARTEN EN EL PLAN DE ESTUDIOS DE LA MAESTRÍA EN AROUITECTURA. CAMPO DE CONOCIMENTO DE TECNOLOGíAs. DE LA unam. EL MÉTODO PERMITE CREAR TECNOLOGIA REAL, PERTINENTE, OPERACIONAL Y VERIFICABLE EXPERIMENTALMENTE EN APLICACIONES ARQUUTECTÓNICAS. LA INFLUENCIA DEL MÉTODO PERMITE UN INCREMENTO DEL INTERÉS Y PERMANENCIA DEL ALUMNO, UN AUMENTO DE LA EFICIENCIA TERMINAL, COMO EL DESARROLLO DE PRODUCTOS DE INTERÉS Y CON POTENCIAL PARA EL PRESENTE Y FUTURO DE LA DISCIPLINA ARQUUTECTÓNICA.

FUENTE: ELABORACIÓN PROPIA COCAMPO RUIZ, 10-11DE JUNIO DE 2019: PONENCIA]. 
realidad muy diferentes objetivos, diferentes lenguajes, diferentes herramientas, diferentes lugares de actuación, diferentes productos, diferentes métodos, como diferentes formas de evaluarlos. Debido a la inconmensurabilidad de visiones y haceres existentes y naturales entre un científico y un tecnólogo, un científico no puede comprender y evaluar correctamente una tecnología, ni sopesar correctamente el trabajo de un tecnólogo o inventor.

Esta situación más que beneficiar el desarrollo tecnológico nacional, lo frenó durante décadas. El CONACYT se dio cuenta de esta situación y a partir de 2014, hizo oficial junto con la Secretaría de Economía, el uso del TRL como método básico para producir, supervisar y evaluar la tecnología nacional en todos los niveles educativos y de investigación en las IEs de la nación. Ahora los pares académicos que evalúan la tecnología son inventores y tecnólogos que han vivido en carne propia la experiencia de investigar y desarrollar tecnología de punta, construyendo prototipos operacionales, plenamente funcionales, probados seriamente en laboratorios y transferidos a la sociedad mediante un proceso de maduración basado en el TRL y una labor de emprendimiento que los hace comercialmente compatibles con la realidad económica e industrial mundial (Sánchez Daza, 2005: 19-20). Permite que una tecnología sea finalmente perfeccionada, incubada y entregada a la sociedad.

Dentro de la disciplina de diseño industrial, y de forma metodológica suprageneral, el TRL marca los pasos correctos de planeación estratégica para las etapas de I\&D+D de Productos Tecnológicos Universitarios, al mismo tiempo que establece un nivel de madurez normalizado y equiparable globalmente.

Para el Diseño Industrial, la herramienta pone orden operacional tanto a los procesos y métodos habituales del diseño (Latorre Cosculluela, 2020: 1-13) para usarse en las etapas de generación creativa de ideas y procesos de materialización de prototipos (Archer, Buchanan, Brown, Cross, Jones, etc.), como al Método Experimental de la Ciencia para generar exclusivamente el espacio experimental de su prueba en cada nivel de maduración que se requiera para alcanzar cualquier tecnología pertinente para nuestra disciplina.

En cada uno de los nueve niveles del método de maduración de tecnología (TRL), al hacer un mejor y más complejo producto, es necesario comenzar con un método de diseño proveniente de cualquier teórico del diseño para generar ideas y construir elementos, componentes y objetos tecnológicos materializados en un prototipo plenamente operacional, para evaluarlos al final de la etapa con una prueba diseñada con el método científico en un espacio experimental confiable y medible (figura 4). Cada etapa requiere de métodos de diseño para empezar y mejorar la tecnología. Cada etapa necesita de una prueba experimental para confirmar y validar el avance en el desarrollo de la tecnología desarrollada. Los métodos de diseño quedan bien ordenados conforme a su papel fundamental durante el proceso de diseño de estos objetos tecnológicos. Su lugar queda no sólo bien articulado con el proceso, sino que no queda duda de por qué y para qué hacerlo. Los modelos tecnológicos han sido probados y esa es su relación y relevancia con la investigación en diseño.

\section{CONCLUSIÓN}

Originalmente tratado en el Foro de Investigación en Diseño en junio de 2019, organizado por el Posgrado de Diseño Industrial de la UNAM (Ocampo Ruiz, 1011 de junio de 2019: Ponencia), frente a participantes de todas las escuelas de diseño nacionales que asistieron, se señaló el aprendizaje análogo que este Posgrado podía adquirir de la experiencia de su hermano, el Posgrado de Arquitectura de la UnAm (Ocampo Ruiz, diciembre de 2019: 5-9), con más de 45 años en el desarrollo de tecnología de punta, epistémica y metodológicamente bien orientada, tal y como lo solicita hoy CONACYT, para beneficiar el trabajo de sus alumnos inscritos en su actual programa y currículo (CONACYT, 2014: 1-7).

Gracias a la diversidad, la amplitud como el alcance temático de los trabajos de investigación y desarrollo de los alumnos del Posgrado del Diseño Industrial de la UNAM, que incluyen temas de tesis que operan en la gama total de incumbencia y pertinencia de todas las disciplinas de diseño, puesto que un alto porcentaje de cada generación que ingresa está integrada por ingenieros, arquitectos, urbanistas, paisajistas, artistas plásticos, diseñadores ambientales, diseñadores de interiores, decoradores, como también diseñadores gráficos e industriales, se considera pertinente la aplicación por analogía de la experiencia y el conocimiento expuestos a cualquier posgrado de diseño nacional o internacional que desarrolle tecnología de punta para estos perfiles temáticos y disciplinarios en su alumnado (tesiunam, 26 de julio de 2019). 


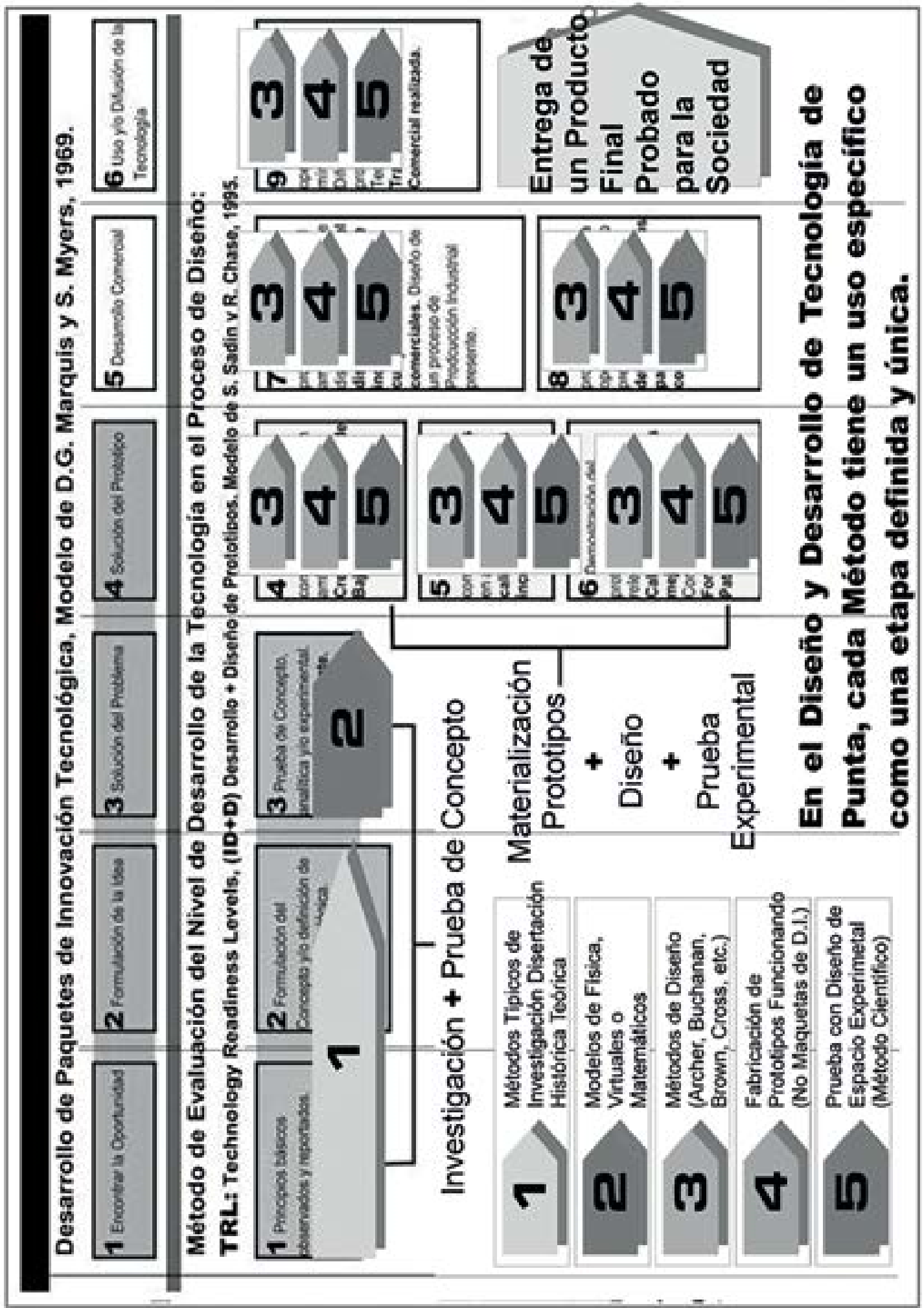

FIGURA 4. INSERCIÓN DE MÉTODOS DE DISEÑO DENTRO DEL TRL. SOBRE LA IMAGEN3, PREVIAMENTE ANALIZADA, SE HA SOBREPUESTO EN CADA ETAPA DE MADURACIÓN DEL TRL: TECHNOLOGY READINESS LEVEL LOS PROCESOS QUE SE NECESITAN EN CADA NIVEL, EN DÓNDE SE REQUUERE DE FORMA EVIDENTE UN MÉTODO DE DISEÑO NECESARIAMENTE PARA LA MEJORA DE LOS PRODUCTOS TECNOLÓGICOS. PARA DIRIGIR TANTO EL PERFECCIONAMIENTO DEL PRODUCTO, A NIVEL ESTÉTICO O FUNCIONAL, COMO PARA MADURARLO COMO PLLOTO COMERCIAL, AL INICIO DE CADA NIVEL DE MADURACIÓN SE REQUUERE LA APLICACIÓN DE UN MÉTODO DE DISEÑO PERTINENTE Y ENFOCADO EN LA SOLUCIÓN DE LOS PENDIENTES A MEJORAR DETECTADOS EN LA ÚLTIMA ETAPA DE PRUEBA. DESPUÉS DE LA APLICACIÓN DEL MÉTODO DE DISEÑO, SE DEBE CONSTRUR UN NUEVO PROTOTIPO, Y DISENÃR UN NUEVO ESPACIO EXPERIMENTAL PARA SU PRUEBA EXITOSA. ESTO SE REPITE POR LO MENOS DESDE EL NIVEL 4 HASTA EL NIVEL 9 DEL TRL, PARA MEJORAR DEL PRODUCTO DE DISEÑO. A NIVEL MACROMETODOLÓGICO, Y SUPRAGENERAL, EL TRL LE DA CERTIDUMBRE Y RAZÓN DE SER A LOS MÉTODOS DE DISEÑO PARA PRODUCIR CORRECTAMENTE OBJETOS TECNOLÓGICOS DE CALIDAD. LES ORDENA, LES DA PROTAGONISMO Y LES HACE VALER SU TRASCENDENTE IMPORTANCIA DENTRO DEL PROCESO. PERO LO QUUE ES MÁS SIGNFICATIVO, PERMITE QUUE UN MÉTODO DE DISEÑO EVOLUCIONE IDEAS CREATIVAS CON RUMMBO DE MATERIALLZACIÓN DEFINIDO, MÁS ALLÁ DE UN TRADICIONAL, SIMPLE Y LIMTTADO MODELO O MAQUETA COMO ENTREGABLE HABITUAL, HACIA EL DESARROLLO DE UN PRODUCTO COMPLEJO PLENAMENTE OPERACIONAL COMERCIALLZABLE, LISTO PARA TRANSFERIRSE A LA SOCIEDAD.

FUENTE: ELABORACIÓN PROPIA COCAMPO RUIZ, 10-11DE JUNIO DE 2019: PONENCIAJ. 
$\mathrm{Al}$ apropiar finalmente esta Visión de Futuro tecnológico, un posgrado de diseño podrá:

1. Fortalecer la producción de conocimiento en la disciplina, como la calidad de la producción tecnológica que se ofrece a la sociedad y que es evaluada en México desde 2014, bajo este enfoque metodológico por CONACYT, que ya incluye oficialmente el Modo 2 de Gibbons más el TRL (CONACYT, abril 2015: 1-7).

2. Permitir el logro de la congruencia entre el objetivo y los productos derivados de la investigación de los temas de los alumnos, frente a la demanda real de soluciones tecnológicas de diseño que se genera afuera de nuestras IEs.

3. Hacer atractivos los proyectos de tesis a los alumnos, mientras se alcanza mejor eficiencia terminal por cohorte generacional, y se reduce la deserción escolar, al ofrecer productos útiles, interesantes, maduros, e inmediatos en su transferencia a la sociedad.

Los beneficios de incorporar estos procesos metodológicos actuales a la investigación en diseño son muchos y de gran valor académico para una IES. Reflexionar acerca de si la investigación en diseño requiere o no de estos métodos particulares instrumentados en los trabajos tecnológicos que realizan los alumnos como tesis para obtener el grado académico, cuando se busca también generar tecnología probada y pertinente, se convierte en trascendente como de importancia estratégica y global, para impulsar y proyectar finalmente por buen camino el futuro de todas las disciplinas de diseño. 


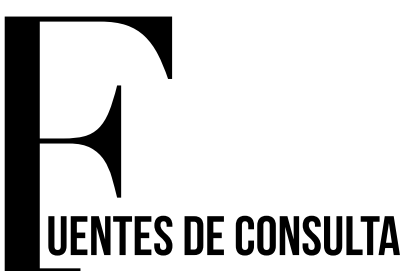

Acuerdo por el que se crea el Programa Transdisciplinario en Investigación y Desarrollo para Facultades y Escuelas, 15 de agosto de 2005. México: Gaceta UNAM, Órgano Informativo de la Universidad Nacional Autónoma de México, p. 24.

Acuerdo por el que se crea la Unidad de Apoyo a la Investigación en Facultades y Escuelas, 15 de agosto de 2005. México: Gaceta UNAM, Órgano Informativo de la Universidad Nacional Autónoma de México, p. 25.

Cadena, G., Castaños, A., Machado, F., Solleiro, J.L., Waissbluth M. (1986), Administración de proyectos de innovación tecnológica, Centro para la Innovación Tecnológica de la Universidad Nacional Autónoma de México, Consejo Nacional de Ciencia y Tecnología, Ediciones Gernika, Ciudad de México.

Casas Guerrero, R. y Valenti, G. (2000), Dos ejes en la vinculación de las universidades en la producción. La formación de recursos humanos y las capacidades de investigación, Instituto de Investigaciones Sociales de la Universidad Nacional Autónoma de México-Universidad Autónoma Metropolitana-Plaza y Valdés Editores, Ciudad de México.

Consejo Nacional de Ciencia y Tecnología (CONACYT) (2014), Programa Marco de Investigación (2014-2020). Anexo 1. TRL o Nivel de Madurez de Tecnología. Consejo Nacional de Ciencia y Tecnología, Ciudad de México.

Consejo Nacional de Ciencia y Tecnología (CONACYT) (2015), Programa Nacional de Posgrados de Calidad (PNPC). Marco de referencia para la evaluación y seguimiento de programas de posgrado presenciales, Consejo Nacional de Ciencia y Tecnología, Ciudad de México.

Didriksson, A. (2005), La universidad de la innovación. Una estrategia de transformación para la construcción de universidades del futuro, Centro de Estudios sobre la Universidad de la Universidad Nacional Autónoma de México-Plaza y Valdés Editores, Ciudad de México.

Estruch Tobella, J. (2002), Dirección profesional y calidad educativa, cIss Praxis, Barcelona.

Gibbons, M., Limoges, C., Nowotny, H., Schwartzman, S., Scott, P., Trow, M. (1997), La nueva producción de conocimiento. La dinámica de la ciencia y la investigación en las sociedades contemporáneas. Ediciones Pomares-Corredor, Barcelona.

Latorre Cosculluela, C., Vázquez Toledo, S., Rodríguez Martínez, A., Liesa Orús, M. (2020), Design Thinking: creatividad y pensamiento crítico en la universidad. México: Revista Electrónica de Investigación Educativa, Instituto de Investigación y Desarrollo Educativo, Universidad Autónoma de Baja California. Volumen 22, e28: 1-13.

López, P, 25 de octubre de 2018. s.v. 117 patentes para la UNAM en 10 años, México: Gaceta UNAM Digital, Órgano Informativo de la Universidad Nacional Autónoma de México. Disponible en http:// www.gaceta.unam.mx/177-patentes-para-la-unam-en-10-anos/, consultado el 25 de julio de 2019.
López, P., 30 de abril de 2018. s.v. La UNAM logró 43 patentes en 2017 México: Gaceta unAm Digital, Órgano Informativo de la Universidad Nacional Autónoma de México. Disponible en http://www.gaceta. unam.mx/20180430/la-unam-logro-43-patentes-en-2017/, consultado el 25 de julio de 2019

Ocampo Ruiz, E., 10-11 de junio de 2019. s.v. Visión de futuro y metodología para la producción moderna de nuevo conocimiento tecnológico en el Posgrado de Diseño Industrial de la UNAM. México: Foro de Investigación en Diseño, Edificio Unidad de Posgrado, Aula B-301, organizado por el Posgrado de Diseño Industrial de la Universidad Nacional Autónoma de México, Ciudad de México: Ponencia presentada.

Roa-Mendoza, C.P. (2016), Investigación en modo 3: Una alternativa para la articulación investigación e intervención en educación superior, Universidad de la Salle, Colombia.

Sánchez Daza, G., Capdeville, M., Corona Treviño, L., Del Valle Rivera, M.C., Jasso Villazul, J., López Leyva, S., Núñez, I. (2005), Innovación en la sociedad del conocimiento. Puebla de los Ángeles, Benemérita Universidad Autónoma de Puebla, Puebla.

Sañudo Guerra, L., Gaeta González, M.L., Soto Bañuelos, E., Segura García, D. (2017), Modos de producción del conocimiento en los programas de posgrado y de formación inicial en educación. El caso de la investigación educativa. San Luis Potosí: XIv Congreso Nacional de Investigación Educativa.

TESIUNAM, Tesis del Sistema Bibliotecario de la Universidad Naciona Autónoma de México. Ciudad de México: Datos de investigación. Disponible en http://www.dgb.unam.mx/index.php/catalogos/ tesiunam, consultado el 26 de julio de 2019.

The Federal Highway Administration, U.S. Department of Transportation (2017), Technology Readiness Level Guidebook. Washington, D.C.: The Federal Highway Administration Exploratory Advanced Research Program.

The United Nations Educational, Scientific and Cultural Organization (UNESCO) (1999), World Conference of Science for the 21st Century: A new Commitment, Budapest, Hungría.

The United States Government Accountability Office, agosto de 2016. Technology Readiness Assessment Guide, Best practices for evaluating the readiness of technology for use in acquisition programs and projects. United States of America: Center for Science, Technology, and Engineering Applied Research and Methods. Document GAO-16$410 \mathrm{G}$.

Unidad de Apoyo a la Investigación para Facultades y Escuelas, Universidad Nacional Autónoma de México (2007). s.v. Dossier de lo Siete Macroproyectos del Programa Transdisciplinario en Investigación y Desarrollo 2005-2007. Unidad de Apoyo a la Investigación para Facultades y Escuelas, Universidad Nacional Autónoma de México, Ciudad de México.

Valle Flores, M.Á., Barrón Tirado, C., Díaz Barriga, F., Elena Marín, D.E., Orozco Fuentes, B., Rigo, M.A., Rojas Moreno, I. (2000), Formación en Competencias y certificación profesional, Centro de Estudios sobre la Universidad de la Universidad Nacional Autónoma de México, México. 